\title{
Person Initials
}

National Cancer Institute

\section{Source}

National Cancer Institute. Person Initials. NCI Thesaurus. Code C94272.

The first letters of the person's first name, middle name, and last name. 